Article

\title{
Morphological Analysis of KDP-Crystal Workpiece Surfaces Machined by Ultra-Precision Fly Cutting
}

\author{
Dongju Chen ${ }^{1}$, Shiwei Zhang, Jingfang Liu *, Chunqing Zha and Ri Pan \\ Beijing Key Laboratory of Advanced Manufacturing Technology, Beijing University of Technology, \\ Beijing 100124, China; djchen@bjut.edu.cn (D.C.); zhangshiwei@emails.bjut.edu.cn (S.Z.); \\ chachunqing@163.com (C.Z.); panri@bjut.edu.cn (R.P.) \\ * Correspondence: jfliu@bjut.edu.cn; Tel.: +86-10-67391619; Fax: +86-10-67391617
}

Received: 12 December 2019; Accepted: 13 January 2020; Published: 16 January 2020 updates

\begin{abstract}
Potassium dihydrogen phosphate crystals exhibit excellent nonlinear optical properties that are significantly affected by the surface morphology of the crystal. To comprehensively examine and characterize the morphological features of these crystals, the machined surfaces of workpieces are analyzed using wavelet, fractal, and power spectral density (PSD) methods. First, the fractal method is employed to analyze the features of the machined surfaces of different materials and examine the relationship between the surface roughness and fractal dimension of different materials. Then, the morphological anisotropy of the machined surfaces is analyzed using the two-dimensional PSD method. Based on the orientation of the machined surfaces of the workpieces, the tangential waves on the surfaces are analyzed using wavelet-transform and PSD methods. From a frequency-domain perspective, the scales of various influencing factor signals are identified. Additionally, the frequency range of the spindle vibration is determined based on the machining experiment. On this basis, the cause of the machined surface waviness errors is revealed.
\end{abstract}

Keywords: potassium dihydrogen phosphate (KDP) crystal; surface morphology; fractal feature; power spectral density

\section{Introduction}

Potassium dihydrogen phosphate (KDP) crystals are a type of nonlinear optical and electro-optical crystal material with exceptional properties. Due to the excellent nonlinear optical properties of these materials, KDP crystals are extensively applied in the components of frequency multipliers, optical modulators, three-dimensional (3D) optical data storage devices, and new-generation 100-joule laser systems [1]. In recent years, the development of solid-state laser technology has led to stricter quality requirements for the machined surfaces of KDP crystals. The surface performance metrics of KDP crystals mainly include form, waviness, and roughness errors [2]. Among them, the waviness error will affect the use of mechanical parts, so the impact of surface waviness has caused many scholars to study this in depth. Adamczak et al. [3] found that an increase in the surface waviness on the inner and outer raceways causes an increase in the vibration level. This effect is most visible for the medium vibration frequency range. Costa et al. [4] addresses the conception of a measurement system to objectively and systematically assess paper superficial waviness in industrial practice. Cao et al. [5] studied the surface waviness of woven ceramic matrix composites and found that surface waviness exerts a tremendous influence on use performance and working life, and the influence degree is dependent on the particular type of surface waviness. For surfaces of high-precision optical workpieces machined by ultra-precision fly cutting, the waviness error of the workpiece surface may cause laser damage to the optical components and directly affect its performance [6]. Therefore, to further improve the 
precision of the machined surfaces of workpieces, waviness errors are an important factor that cannot be overlooked.

Many factors will affect the morphological characteristics of the workpiece, such as mechanical vibration [7], crystal orientation [8], material physical properties [9], and spindle speed [10]. Chi et al. [11] studied the effects of blade tip vibrations, material properties, and spindle rotation errors on ultra-precision diamond-turned surfaces through multispectral analysis. Chen et al. [12] determined the effects of the frequency response of the spindle on frequency-domain errors of machined surfaces by analyzing aerostatic spindle errors. An et al. [13] examined the effects of an unbalanced aerostatic spindle on the machined surfaces of workpieces and found that the waviness errors caused by the unbalanced spindle were less than $0.1 \mu \mathrm{m}$ and the wavelength was approximately $100 \mathrm{~nm}$. Based on the kinematic principle of planar milling, Baek et al. [14] established a surface roughness simulation model and used the model to study the effects of radial runout errors and feed speed on machined surfaces; these researchers found a strong nonlinear relationship between the feed speed and surface roughness. Through experiments on diamond turning of an aluminum alloy (Al 6061) and a single-crystal Al, Cheung et al. [15] studied the effects of the material properties on the formation of the surface microstructure of the workpieces. However, these studies primarily focused on the effects of various factors on the surface morphology, and there is a lack of systematic research on the surface morphology of KDP crystals.

To comprehensively analyze and characterize the morphology of the machined surfaces of workpieces, the machined surfaces of the KDP crystal are analyzed using wavelet, fractal, and power spectral density (PSD) methods. First, the fractal method is used to determine the 3D fractal dimension (Ds) of the machined surfaces of various materials. Then, the surface roughness-Ds relationship is analyzed for each material. Additionally, the fly-cutting-machined KDP crystal surface is found to exhibit high anisotropy using the two-dimensional (2D) PSD method. Based on the orientation of the machined surfaces of the workpieces, the tangential waves on the surfaces are analyzed using the wavelet-transform method. From a frequency-domain perspective, the scale of each influencing factor signal is identified. The PSDs are analyzed and compared with the simulated values. On this basis, the frequency range of the spindle vibrations is identified. Furthermore, the cause of the machined surface waviness errors is revealed.

\section{The Structure of KDP Crystal and the Analysis Method of Surface Morphology}

\subsection{The Structure of KDP Crystal and Crystalline Orientation}

KDP crystals belong to a tetragonal crystal system at room temperature, with the $\mathrm{D}_{2 \mathrm{~d}}-42 \mathrm{~m}$ point group and the $\mathrm{D}^{12}{ }_{2 \mathrm{~d}}-\mathrm{I} 42 \mathrm{~d}$ space group. The unit cell parameters are $\mathrm{a}=\mathrm{b}=0.7453 \mathrm{~nm}, \mathrm{c}=0.6975 \mathrm{~nm}$, and $Z=4$. The cell structure of the KDP crystal is shown in Figure 1a. Its ideal shape is one formed by the aggregation of a quadrangular column and a quadrangular double cone, as shown in Figure $1 \mathrm{~b}$. The structure of the KDP crystal determines its properties. A KDP crystal is an anisotropic material with strong anisotropy in physical and mechanical properties.

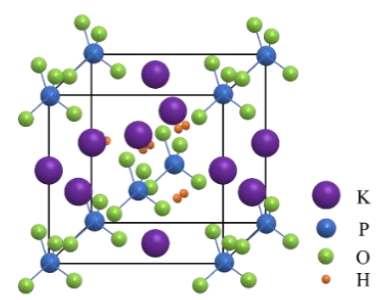

(a)

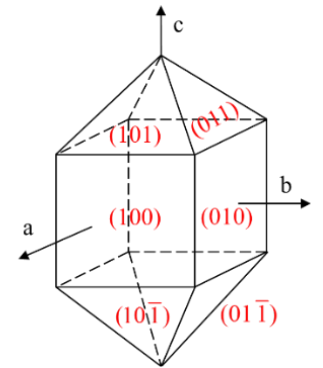

(b)

Figure 1. The structure of a KDP (potassium dihydrogen phosphate) crystal: (a) The cell structure of a KDP crystal; (b) The ideal shape of a KDP crystal. 
A schematic diagram of the crystal orientation of the KDP crystal is shown in Figure 2. The plane ABCD is the (001) crystal plane. The directions of AD, AC and AB are the crystalline directions of [100], [110] and [010], respectively. The crystalline directions of [100], [110] and [010] are defined as 0, 45 and 90 crystalline orientation, respectively.

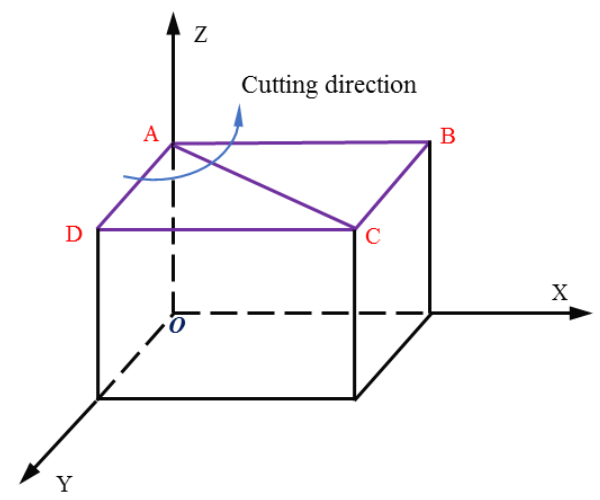

Figure 2. The crystalline orientation of the KDP crystal.

\subsection{Morphological Orientation of Surfaces}

If the physical properties of the material of a workpiece are consistent in various directions, then the physical properties of the material are unrelated to the orientation, and the material is isotropic; otherwise, the material is anisotropic. Like the physical properties of the workpiece material, the topological structure of the workpiece surface is also isotropic and anisotropic. The isotropic workpiece surface has the same topological structure in all directions, while the anisotropic workpiece surface may have a different topological structure in different directions.

Based on the properties of surfaces, Thomas categorized anisotropic surfaces into weakly and strongly anisotropic surfaces [16]. The profile information of a weakly anisotropic workpiece surface is different in each direction, and the profile information in any direction plays a major role on the entire surface of the workpiece. For a strongly anisotropic workpiece surface, the profile information of only one direction plays a major role for the entire surface of the material, whereas the profile information of all the other directions can be treated as the superposition of the profile information in this direction along the orthogonal direction. Therefore, when analyzing strongly anisotropic machined surfaces, it can be simplified by analyzing the surface profile information in one direction. Ds can comprehensively reflect the surface topology of a workpiece and thus can be used to study the orientation of the machined surface of a workpiece.

\subsection{Fundamental Theory of the Fractal Method}

On a microscopic scale, the surface of the workpiece is rough and not smooth. Surface profiling shows that on a microscopic scale, as magnification increases, a surface profile does not tend to be a straight line but instead maintains an approximately similar shape. The state where a part of an object is similar to the whole in a certain way is called fractal, and the fractal geometry has self-similarity and scale invariance [17]. The fundamental principle of the fractal method is as follows,

$$
D=\frac{\ln N}{\ln S}
$$

where $D$ is the theoretical fractal dimension and $N$ and $s$ are scale parameters related to self-similar features. 


\subsection{Calculation of Ds}

Ds is a minor modification of the conventional concept of dimensions. This modification allows the concept of non-integer dimensions. Ds is the most important characterization parameter in fractal theory. This parameter reflects the microscopic morphological features of a surface. The more complex the microscopic morphology is, the greater the Ds is. Box counting is a method commonly used to calculate $D_{s}$. This method can be used to perform calculations on curves or areas encircled by curves, e.g., surface morphology, pore distribution, and crack propagation. Box-counting dimension can be represented by the following,

$$
D_{s}=\lim _{k \rightarrow \infty} \frac{\ln N_{\delta_{k}}(F)}{-\ln \delta_{k}}
$$

where $D_{s}$ is the fractal dimension, $\delta$ is the length of squares, $F$ is the arbitrary non-empty bounded subset of space and $N_{\delta_{k}}$ is the number of $\delta_{k}$ grids intersecting $F$.

The box-counting method is also called the pixel-counting method because this method requires the use of $2 \mathrm{D}$ square lattices with different side lengths $\left(\delta_{k}\right)$ to calculate the numbers $\left(N_{\delta_{k}}(F)\right)$ of square lattices with different $\delta_{k}$ needed to cover $F$. Additionally, this method uses the least-squares approach to perform a unary linear regression on $\ln \delta_{k}$ and $\ln N_{\delta_{k}}(F)$. Thereby, the value of $D s$ is obtained. This method agrees with the fundamental definition of fractal theory and can be easily implemented by computer programming. Thus, the box-counting method is employed in this study to calculate the $D_{\mathcal{S}}$ of the surface profiles of the workpieces.

\section{Results and Discussion}

\subsection{Experimental Results}

First, a KDP crystal plane with a diameter of $410 \mathrm{~mm}$ is machined on an ultra-precision fly-cutting lathe, with the machining parameters are shown in Table 1 . This lathe is primarily used to machine large-diameter planar optical components and is capable of semifinished and finished machining of special hard and brittle materials such as KDP crystals. Combining the linear feed motion of the worktable with the rotation motion of the spindle, the single point diamond ultra-precision flying tool cutting of large-aperture optical planar elements can be realized. A WYKO NT9300 optical profiler (Veeco Instruments Inc., Plainview, NY, USA) is used to examine the form accuracy of the machined workpiece. Table 2 shows the measurement parameters of the measuring system. Figure 3 shows the experimental result. Figure 3a shows the results of the machined surface, and it can be seen that there is a significant texture on the surface. For further observation, a $25 \mathrm{~mm}$-wide area outside the range of the cutting edge (the red area in Figure $3 a$ ) is extracted, as shown in Figure $3 b$. Figure $3 c$ shows the 2D morphology of the surface in the cutting direction. As demonstrated in Figure $3 b, c$, notable waviness with a spatial period of $9.4 \mathrm{~mm}$ and an amplitude of approximately $24.9 \mathrm{~nm}$ is present on the machined surface along the cutting direction. The rotational speed (n) of the spindle is $300 \mathrm{rpm}$, and the radius $(\mathrm{R})$ of the fly-cutter head is $315 \mathrm{~mm}$. On this basis, the conversion space period to the time-frequency domain is about $526 \mathrm{~Hz}$.

Table 1. Parameters for surface machining.

\begin{tabular}{cc}
\hline Parameters & Values \\
\hline Spindle speed $n(\mathrm{rpm})$ & 300 \\
Feed rate $f(\mu \mathrm{m} / \mathrm{s})$ & 30 \\
Cutting depth $a_{p}(\mu \mathrm{m})$ & 4 \\
Tool rake angle $\left(^{\circ}\right)$ & 45 \\
Tool radial error $(\mathrm{xS}(\mu \mathrm{m}))$ & 10 \\
Tool error angle $\beta\left({ }^{\circ}\right)$ & 0.5 \\
Radius of cutter head $R(\mathrm{~mm})$ & 315 \\
Workpiece width $W(\mathrm{~mm})$ & 410 \\
\hline
\end{tabular}


Table 2. Measurement parameters of the measuring system.

\begin{tabular}{cc}
\hline Parameters & Values \\
\hline Sampling interval & $0.01 \mathrm{~ms}$ \\
Roughness RMS repeatability & $0.005 \mathrm{~nm}$ \\
Measuring range & $14 \mathrm{~mm} \times 10 \mathrm{~mm}$ \\
Kind of filter & $0.1 \mathrm{~nm}$ \\
Z-direction resolution & remove shape standard filtering filter spectrum \\
\hline
\end{tabular}

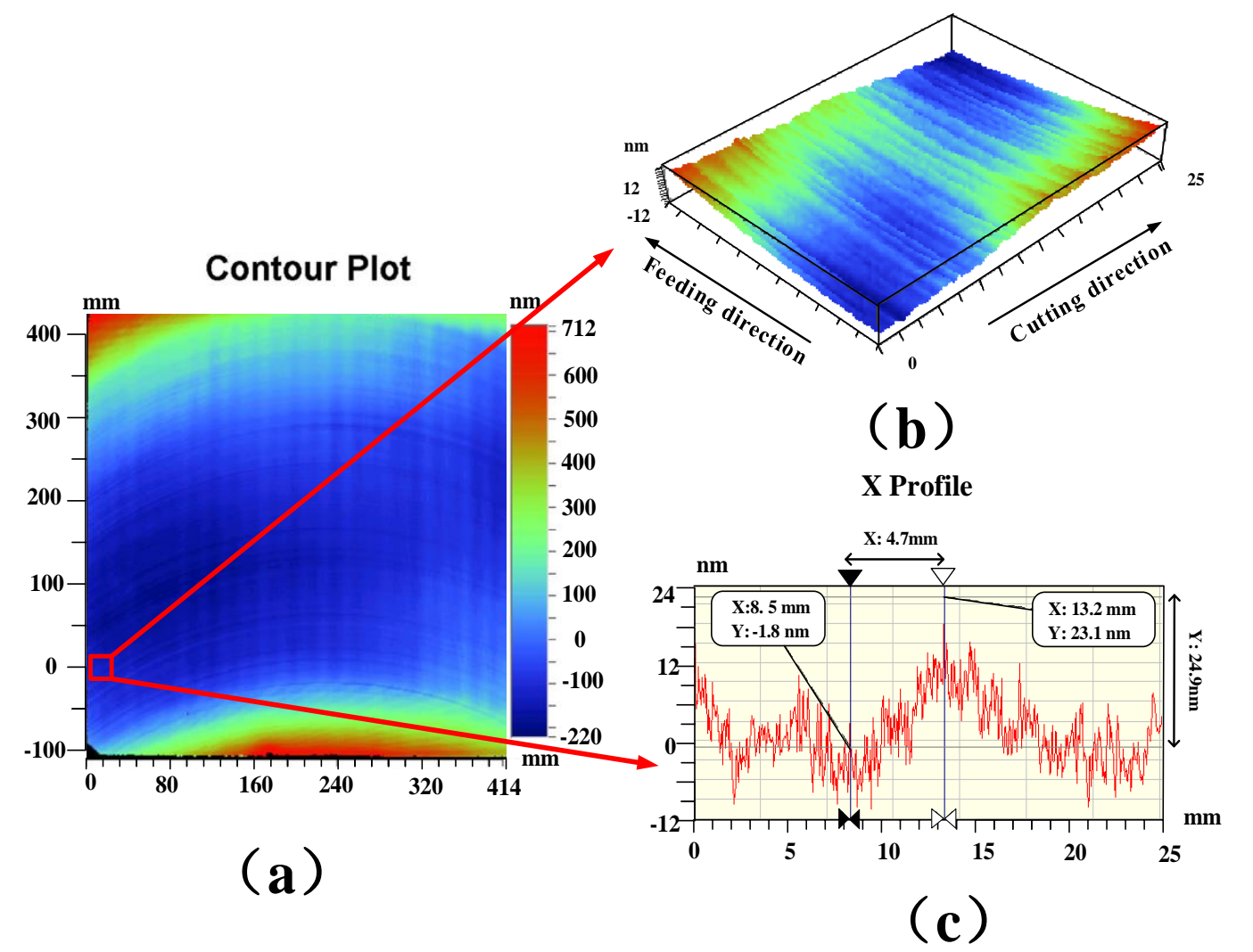

Figure 3. Machining surface results: (a) experimental results; (b) sample surface; (c) surface profile.

In order to study the directional characteristics of the surface of ultra-precision machining workpieces, ultra-precision fly-cutting machine tools are used to cut Al-alloy and KDP-crystal materials. During the machining process, the spindle speed is $200 \mathrm{rpm}$, the feed speed of the workbench is $30 \mu \mathrm{m} / \mathrm{s}$, and the cutting depth is $4 \mu \mathrm{m}$. A total of six groups of data are measured. Due to the limited length of this article, sectional profiles are drawn based on the first three groups of data. Figures 4 and 5 show the profiles of certain sections of the surfaces of the Al-alloy workpiece and the KDP-crystal workpiece, respectively. 

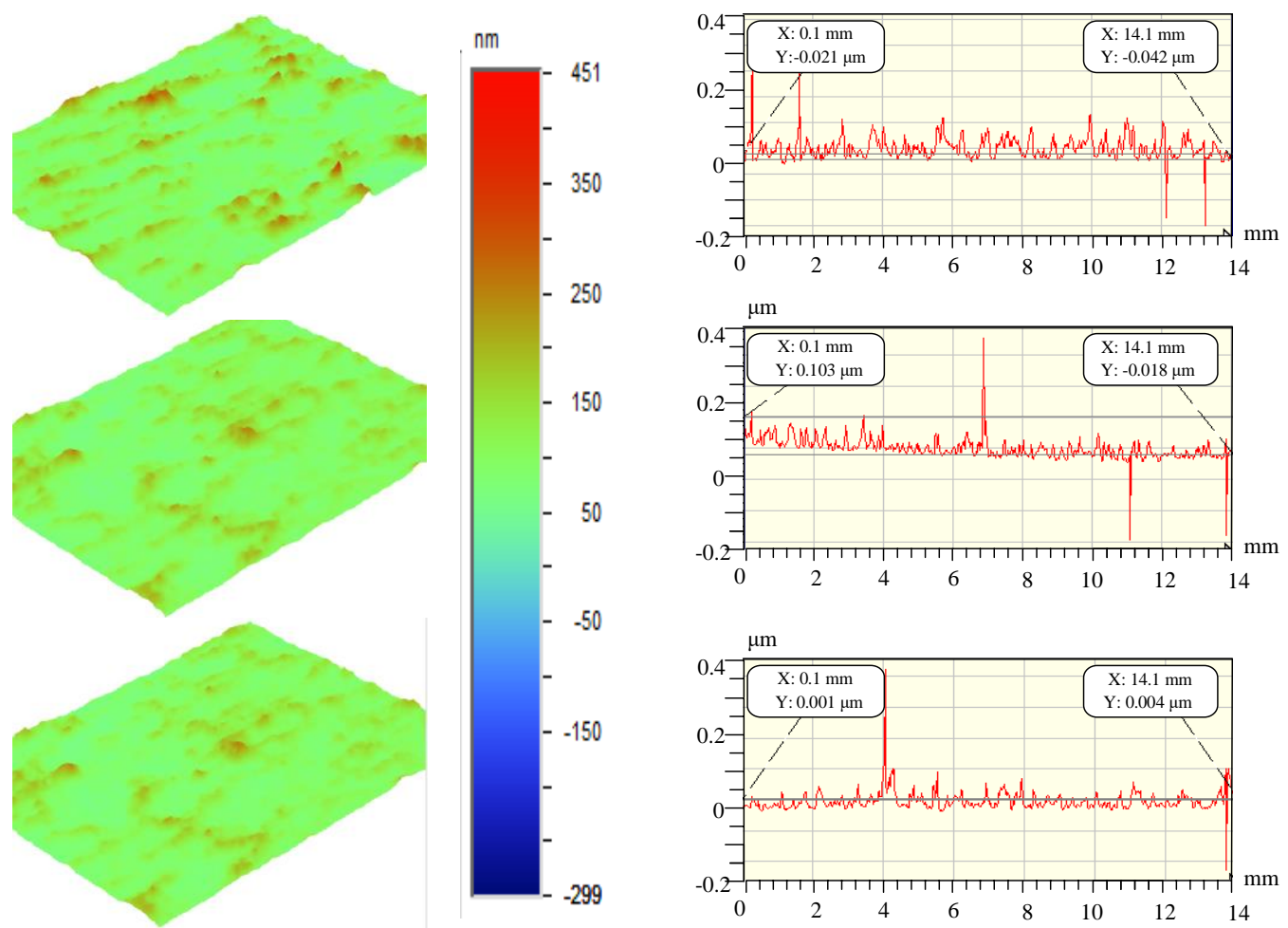

Figure 4. Al alloy surface profile.
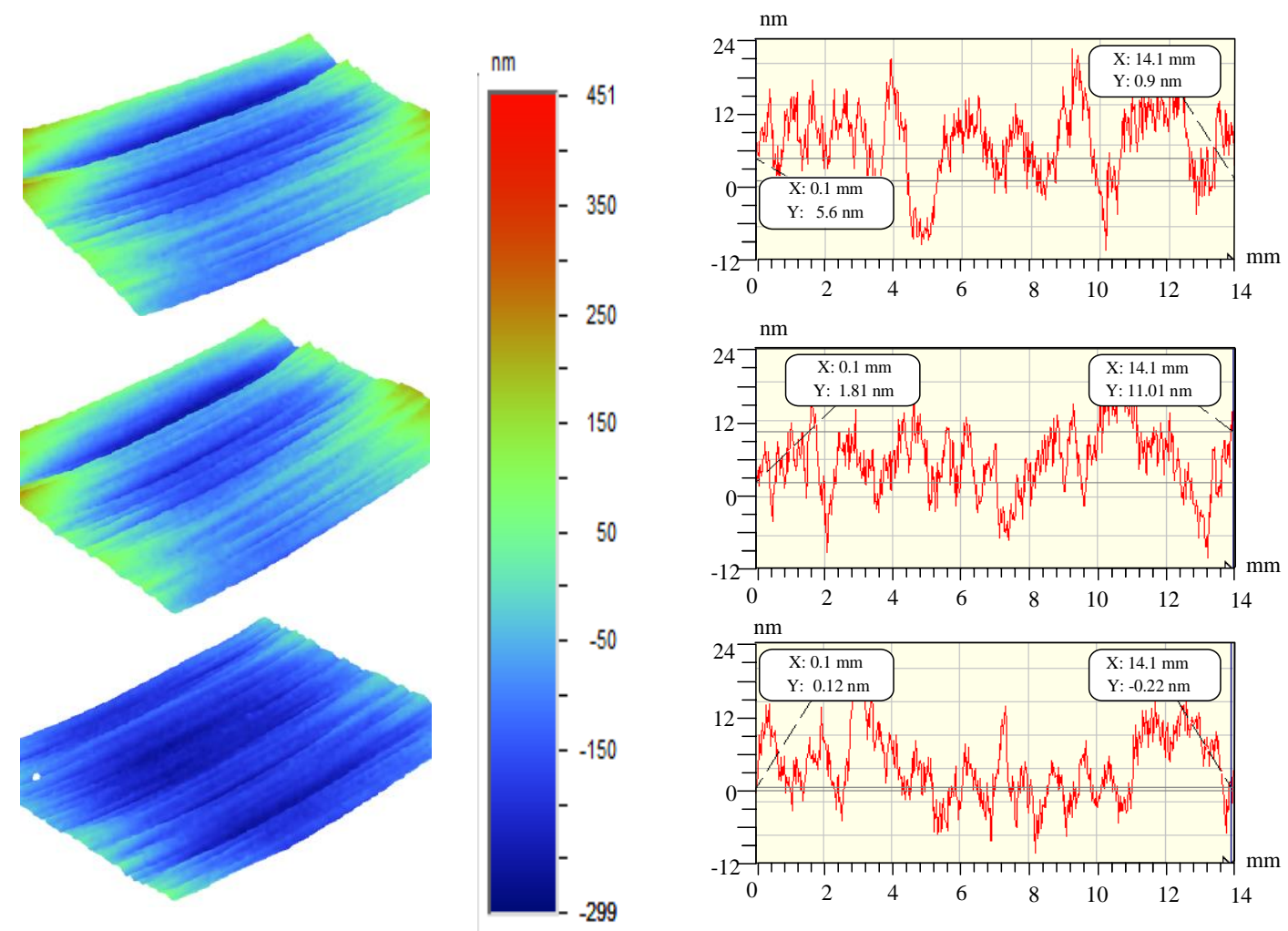

Figure 5. KDP crystal surface profile.

\subsection{Fractal Analysis of the Machined Surfaces}

In order to study the differences in the morphology of the workpiece surfaces represented in Figures 4 and 5, the three-dimensional fractal dimension Ds of the surface data for each workpiece is 
calculated in MATLAB (MATLAB R2016a, MathWorks, Natick, MA, America) using Equation (2) (i.e., the box-counting method), introduced in Section 2.4. Table 3 summarizes the Ds values. Based on the analysis of Ds in Section 2.4 and Table 3, the 3D Ds of the surface of the Al-alloy workpiece is mostly smaller than that of the surface of the KDP-crystal workpiece, which indicates that the surface of the KDP-crystal workpiece has a more complex microstructure.

Table 3. Fractal dimension (Ds) of the workpiece surface.

\begin{tabular}{ccccccc}
\hline \multirow{2}{*}{ Material } & \multicolumn{7}{c}{$\boldsymbol{D}_{\text {s }}$} \\
\cline { 2 - 7 } & $\mathbf{1}$ & $\mathbf{2}$ & $\mathbf{3}$ & $\mathbf{4}$ & $\mathbf{5}$ & $\mathbf{6}$ \\
\hline Al alloy & 2.37 & 2.35 & 2.34 & 2.35 & 2.39 & 2.36 \\
KDP crystal & 2.71 & 2.75 & 2.68 & 2.73 & 2.69 & 2.71 \\
\hline
\end{tabular}

Comparing the experimental results in Figures 4 and 5 and the three-dimensional fractal dimensions in Table 3, it can be seen that the surfaces machined by fly cutting conform to the $D_{s}$ features. The finer a surface is, the higher the space-filling capacity of the surface is, and the higher the $D_{s}$ of the surface is. As demonstrated by the calculated data in Table 3, the $D_{s}$ associated with ultra-precision fly cutting generally ranged from 2.34 to 2.73 , which indicates that the surfaces machined by fly cutting have a relatively complex morphological structure.

To validate the calculated values of $D_{s}$ in Table 3 , a corresponding parameter $R_{q}$ (root-mean-square surface roughness) that characterizes the 3D-machined surface morphology was calculated. For discretely sampled data, $R_{q}$ can be calculated using the following equation,

$$
R_{q}=\sqrt{\frac{1}{l_{x} l_{y}} \int_{0}^{l_{y}} \int_{0}^{l_{x}} z^{2}(x, y) d x d y}=\sqrt{\frac{1}{M N} \sum_{j=1}^{N} \sum_{i=1}^{M} z^{2}\left(x_{i}, y_{j}\right)}
$$

where, $z(x, y)$ is the profile of surface, $l_{x}$ and $l_{y}$ are the side length of the sampling area and $M$ and $N$ are the number of discrete samples in the $x$ and $y$ direction within the sampling interval.

The measuring range of the WYKO NT9300 optical profiler on the two surfaces was $14 \mathrm{~mm} \times 10 \mathrm{~mm}$. There was a total of $640 \times 480$ sampling points. The $R_{q}$ of the machined workpieces in Figures 4 and 5 was calculated using Equation (3). Table 4 summarizes the results. Based on Table 4 , combined with the 3D $D_{s}$ of the machined surfaces in Table 1 , the $R_{q}$ of the Al-alloy workpiece was higher than that of the KDP-crystal workpiece. However, the $3 \mathrm{D} D_{s}$ of the Al-alloy workpiece was mostly lower than that of the KDP-crystal workpiece.

Table 4. Workpiece surface roughness $\left(R_{q}\right)$.

\begin{tabular}{ccccccc}
\hline \multirow{2}{*}{ Material } & \multicolumn{7}{c}{$\boldsymbol{R}_{\boldsymbol{q}}$} \\
\cline { 2 - 7 } & $\mathbf{1}$ & $\mathbf{2}$ & $\mathbf{3}$ & $\mathbf{4}$ & $\mathbf{5}$ & $\mathbf{6}$ \\
\hline Al alloy & $45.47 \mathrm{~nm}$ & $48.02 \mathrm{~nm}$ & $48.57 \mathrm{~nm}$ & $44.27 \mathrm{~nm}$ & $43.15 \mathrm{~nm}$ & $46.23 \mathrm{~nm}$ \\
KDP crystal & $10.68 \mathrm{~nm}$ & $9.36 \mathrm{~nm}$ & $13.26 \mathrm{~nm}$ & $10.86 \mathrm{~nm}$ & $12.54 \mathrm{~nm}$ & $11.63 \mathrm{~nm}$ \\
\hline
\end{tabular}

In order to analyze the relationship between the surface roughness of the workpiece and the three-dimensional fractal dimension, the $R_{q}-D_{s}$ curves are plotted for the workpieces made of different materials based on the data in Tables 3 and 4, as shown in Figure 6. As demonstrated in Figure 6, the $D_{S}$ of the surface of the workpiece basically decreases as $R_{q}$ increases. This result indicates that the $D_{S}$ values calculated in this study are accurate, thereby further demonstrating the theory that the greater the $D_{s}$ is, the finer the microscopic topology is. To further examine the relationship between $R_{q}$ and $D_{s}$, a $D_{s}$ prediction model is established based on a backpropagation (BP) neural network. 


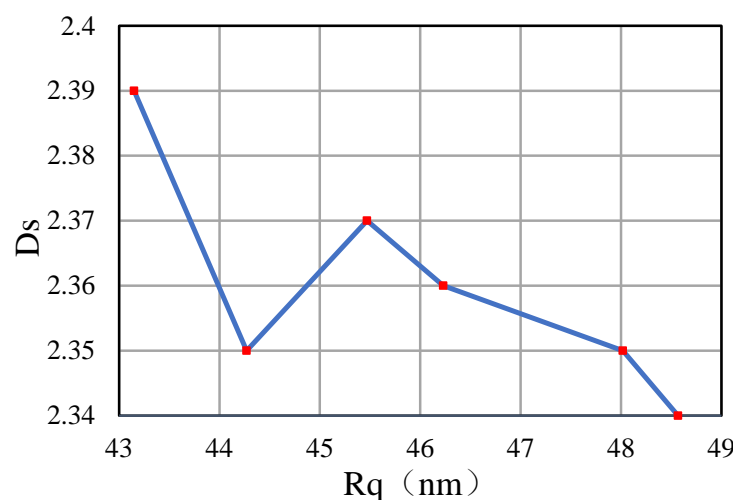

(a)

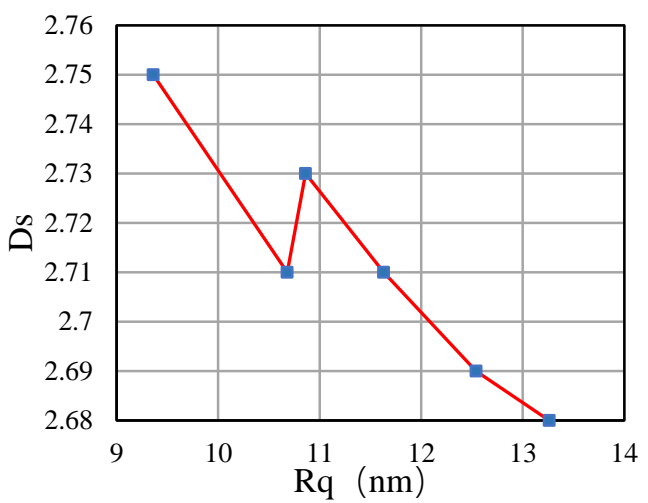

(b)

Figure 6. Relationship between surface roughness and fractal dimension: (a) Al alloy; (b) KDP crystal.

\subsection{Ds Prediction Model}

A BP neural network is a multilayer feedforward neural network based on the error BP algorithm. A BP neural network describes the structure of a biological neural network with a certain simple mathematical model, and under certain algorithms, it can simulate the intelligent behavior of a biological neural network to some extent, so as to solve the problem of intelligent information processing which traditional algorithms cannot compete for. The network model is shown in Figure 7. Compared with traditional statistical methods, the BP neural network has the following advantages: it does not require the type or distribution of materials, it does not need to define the mathematical equation of the mapping relationship between input variables and output variables beforehand, and it can define the complex mapping relationship between them through self-learning and self-organization, so it has a good ability to deal with non-linear problems. Therefore, this paper chooses a BP neural network to establish the prediction model of the fractal dimension of the workpiece surface.

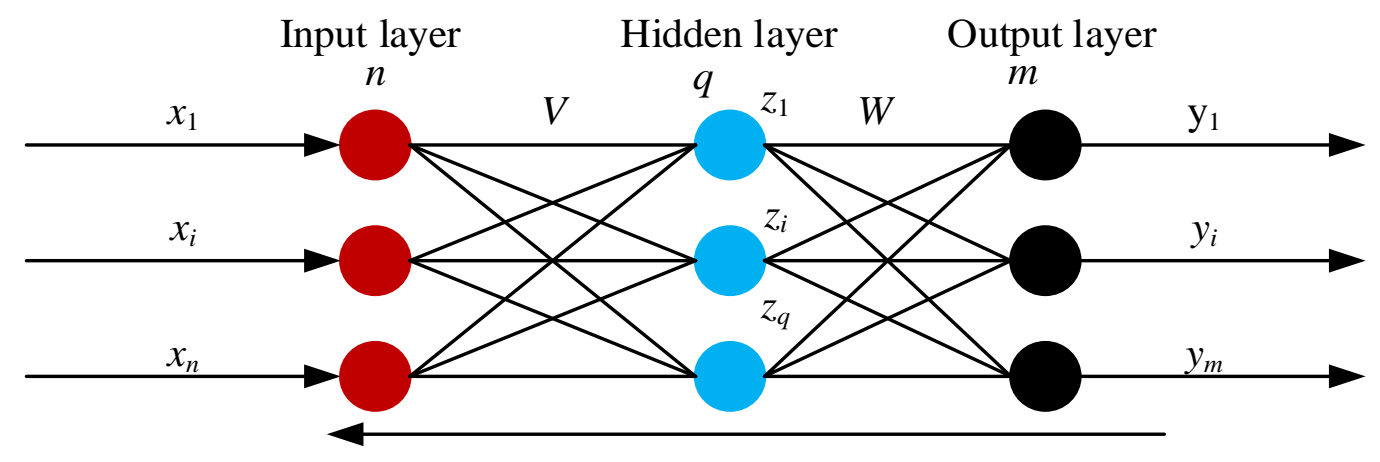

Error back propagation

Figure 7. Network model.

In order to improve the training accuracy of the BP neural network calculation, this paper normalizes all the data, and then carries on the inverse normalization after the training. The prediction model of the fractal dimension of the workpiece surface is established based on the BP neural network, and the modeling process is completed with the assist of the neural network toolbox of MATLAB. In this network, $R_{q}$ and $D_{s}$ are the input and output ends, respectively. The number of neurons in the hidden layer is calculated using an empirical equation.

$$
n=\sqrt{\text { num_input }+ \text { num_output }}+a
$$

Where, $n$ is the number of neurons in the hidden layer, num_input is the number of input neurons, num_output is the number of output neurons and $a$ is the constant between 1 and 10 . 
Based on Equation (4) combined with the actual conditions, the network model established in this study has a relatively high prediction accuracy and convergence rate when there are eight neurons in the hidden layer. According to the fractal dimension of the surfaces of the machined workpieces in Figure 6, the prediction curves of the $R_{q}$ and $D_{s}$ of the surfaces of the machined workpieces are shown in Figure 8. Figure 8a shows the measured and predicted values of the $R_{q}$ and $D_{s}$ of the surface of the Al-alloy workpiece. Figure $8 \mathrm{~b}$ shows the measured and predicted values of the $R_{q}$ and $D_{s}$ of the surface of the KDP-crystal workpiece. In Figure 8, the blue curves are based on the experimental data in Figure 6. The prediction model generated by training the network was capable of predicting the $D_{S}$ for other $R_{q}$ values, as shown by the red curves. The predicted curves were found to be very close to the measured experimental curves, demonstrating a very high prediction accuracy.

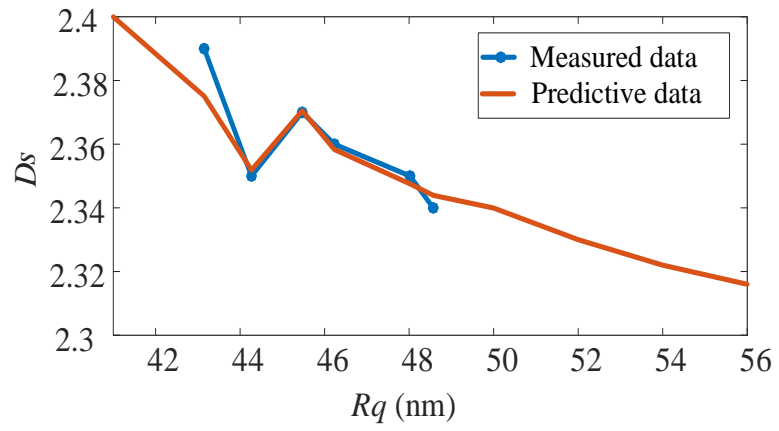

(a)

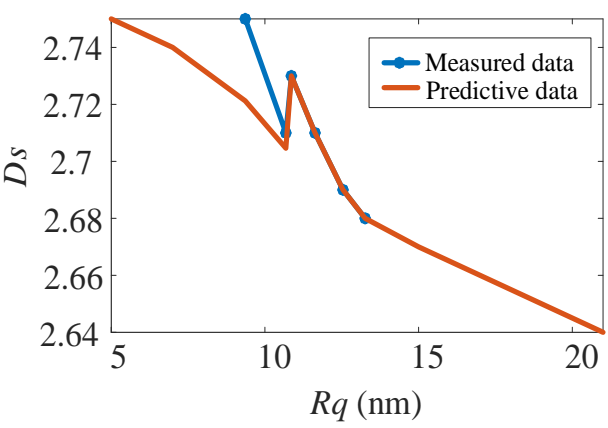

(b)

Figure 8. Prediction model of surface fractal dimension: (a) Al alloy; (b) KDP crystal.

As demonstrated in Figure 8, the $R_{q}$ of each ultra-precision machined surface was essentially inversely proportion to the corresponding $D s$. That is, the lower the surface roughness of the workpiece, the bigger its fractal dimension. However, there was no strict corresponding relationship between $D_{S}$ and $R_{q}$. As demonstrated in Figure $8 \mathrm{a}$, the $R_{q}$ of the Al-alloy workpiece ranged from 44 to 46 , and $D_{s}$ was proportional to $R_{q}$. This result suggests that there is no strict, direct relationship between the $R_{q}$ and $D_{s}$ of a workpiece; that is, $D_{s}$ is a surface characterization parameter that is independent of $R_{q}$. In Figure $8 \mathrm{a}$, the $R_{q}$ of the surface morphology of the $\mathrm{Al}$ alloy ranges from 42 to 44 . The surface roughness is the same, but the fractal dimension is quite different. It can be further inferred that in the process of ultra-precision machining, the roughness of the machined surface can be kept unchanged by reasonable selection, while the surface microstructures change. This conclusion is of great significance for the study of optical elements. Therefore, our further research direction is to study the influence of cutting parameters on fractal features. It mainly analyzes the fractal characteristics of surface morphology, and the influence of various cutting parameters on surface roughness is not the focus of consideration. Only by fully understanding the influence of processing parameters on the fractal characteristics can we formulate reasonable processing parameters to optimize the surface quality of the workpiece and achieve the purpose of improving its optical performance.

\subsection{Fractal Analysis of Surface Morphology Anisotropy}

As mentioned in Section 2, similar to the physical properties of the material, the surface topology of a workpiece can be isotropic or anisotropic. When processing an anisotropic material, as the cutting direction of the tool changes with respect to the crystal orientation of the crystal, the deformation generated inside the crystal material also changes. When the material is plastically deformed, a smooth region is formed on the surface of the workpiece. When a brittle fracture occurs, a fracture failure zone is formed, which seriously affects the surface quality of the workpiece. The above phenomenon will cause anisotropy on the surface of the machined workpiece. Therefore, for the anisotropic materials, the $R_{q}$ and $D_{s}$ of the material vary in different directions. As a result, the statistical properties also vary in different directions. It can be seen from Figure 8 that even if the $R_{q}$ of a certain section of 
the workpiece surface morphology is the same, its $D_{s}$ may be different. Thus, to comprehensively analyze the surface information, the $D_{s}$ of the surface of each machined workpiece in various directions is analyzed.

Due to the limited length of this article, for the different materials, only a set of experimental data is selected to draw the $D_{s}$ of the surface profile in different directions, as shown in Figure 9. Figure $9 \mathrm{~b}$ shows the $D_{s}$ of the surface profile of the KDP-crystal workpiece. The $D_{s}$ values of the surface profiles of this workpiece are mostly greater than 1.5, suggesting that the KDP-crystal workpiece has a relatively complex surface morphology. Further observation of Figure 9a,b shows that there is no significant fluctuation in the $D_{s}$ of the surface profile of the workpiece in Figure 9a in various directions, while the surface profiles of the workpiece in Figure $9 \mathrm{~b}$ have a small fractal dimension of the surface contour in the $90^{\circ}$ and $270^{\circ}$ directions. As a result, the $D_{s}$ values of the circumferential profiles are more symmetrical in Figure $9 \mathrm{~b}$ than in Figure $9 \mathrm{a}$. The $D_{s}$ values in the $90^{\circ}$ and $270^{\circ}$ directions differ relatively significantly from those in other directions. This result also suggests that the profile features in the direction parallel to the $90^{\circ}$ or $270^{\circ}$ directions play a main role for the entire surface of the workpiece. Based on Thomas' theory introduced in Section 2.2, the machined surface of this workpiece is anisotropic, indicating that the profiles parallel to the cutting direction are the main features of this surface.

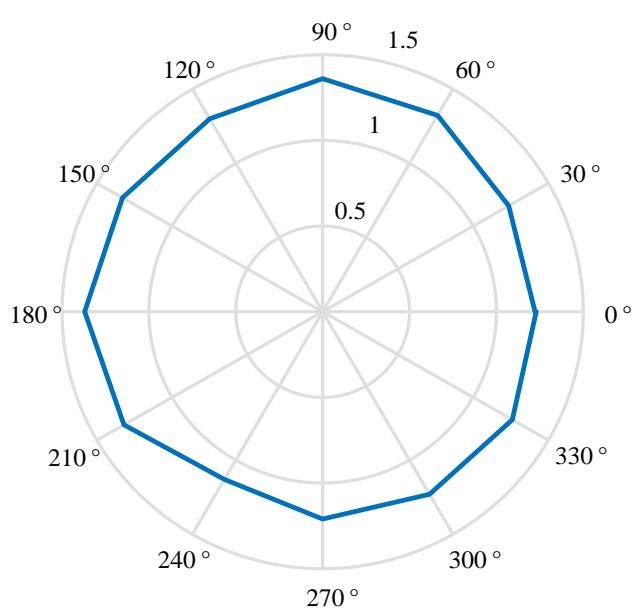

(a)

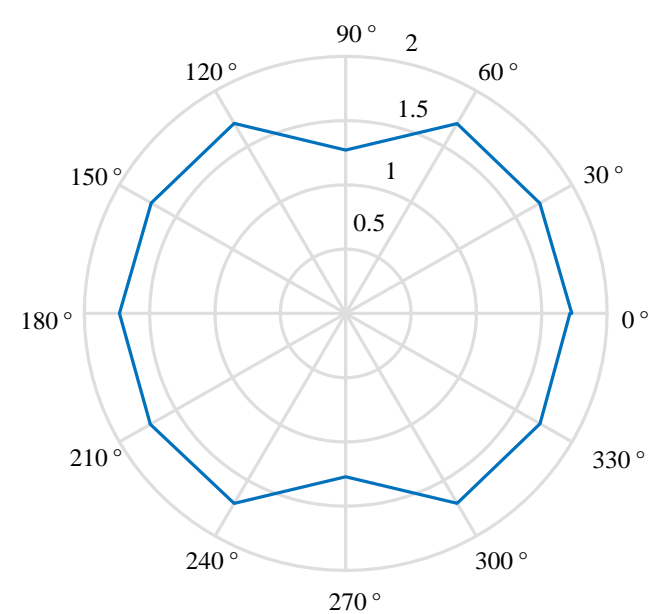

(b)

Figure 9. Fractal dimension of surface profile: (a) Al alloy; (b) KDP crystal.

\section{2D Power Spectrum Analysis}

The power spectral density is established based on the analysis of the structure, so the power spectral density of the machined surface of the KDP crystal should be consistent with the fractal characteristics to a certain extent. To verify the fractal features of the surface of the KDP-crystal workpiece discussed in Section 3, the 2D PSD analysis method is employed. PSD can be derived from the fast Fourier transform (FFT) theory.

Performing a 2D FFT can be treated as processing two continuous one-dimensional (1D) FFTs. In practice, the surface of a workpiece is characterized based on a finite number of digital sampling points. Therefore, a 2D discrete FFT is often used for the numerical calculations in practice.

$$
G\left(\frac{u}{M T_{x}}, \frac{v}{N T_{y}}\right)=\sum_{q=0}^{N-1} \sum_{p=0}^{M-1} z\left(p T_{x}, \mathrm{q} T_{y}\right) e^{-j 2 \pi\left|\frac{u}{M} p+\frac{v}{N} q\right|}
$$

where $M$ and $N$ are the sampling points, and $T_{x}$ and $T_{y}$ are the sampling length. 
In this study, the direct periodogram method is used to estimate the 2D PSD of the surface, and it is defined as follows:

$$
G\left(\frac{u}{M T_{x}}, \frac{v}{N T_{y}}\right)=\frac{1}{M N T_{x} T_{y}}|H| \frac{u}{M T_{x}}, \frac{v}{N T_{y}} \|^{2}
$$

where $|H| \frac{u}{M T_{x}}, \frac{v}{N T_{y}} \|$ is the amplitude-frequency characteristics and $G\left(\frac{u}{M T_{x}}, \frac{v}{N T_{y}}\right), \frac{1}{M N T_{x} T_{y}}$ is the sequence length.

When used to estimate spectra, the periodogram method can easily result in PSD energy leakage. Previous studies have shown that a data window can be used to effectively avoid this problem. Based on the above theory, a two-dimensional power spectral density analysis on the surface of KDP crystals and aluminum alloys processed by ultra-precision fly cutting is performed, as shown in Figure 10. Only one frequency-domain spectrum is present in the PSD diagram of the machined surface of the Al-alloy workpiece. The energy of the fly-cutting-machined surface of the KDP-crystal workpiece is mainly concentrated in the direction vertical to the surface texture. The machined surface is mainly affected by its spatial frequency in the direction vertical to the cutting direction. This result agrees with the finding obtained using the fractal method for 2D circumferential profiles-the features of the machined surface of the KDP-crystal workpiece consisted mainly of the profiles parallel to the cutting direction. Additionally, because the $D_{s}$ values of the profiles parallel to the cutting direction are relatively small, the PSD is insignificant in the direction parallel to the cutting direction. The analytical results for the PSD are consistent with those for the Ds of the circumferential surface profiles. This result demonstrates that fractal analysis can reflect not only the microstructure and anisotropy of a surface but also, to a certain extent, the spectral frequency distribution of the surface.

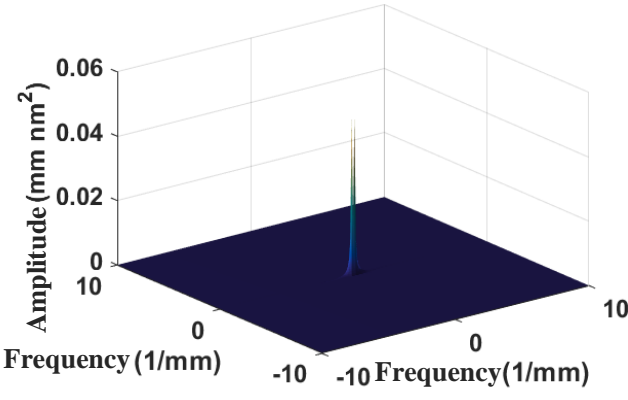

(a)

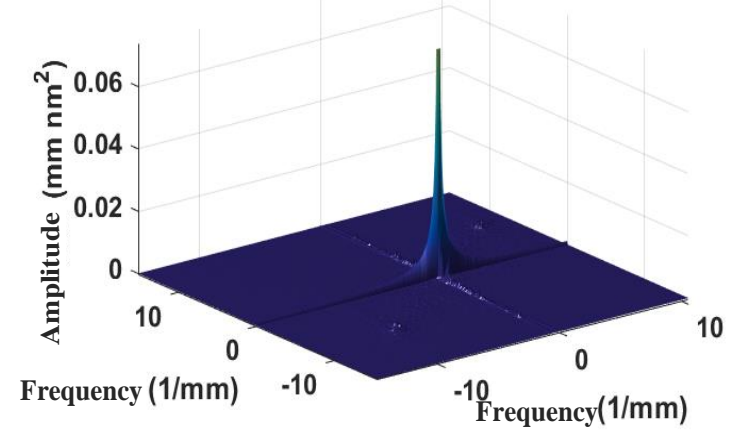

(b)

Figure 10. Surface power spectral density: (a) Al alloy; (b) KDP crystal.

Based on the above analysis, the fly-cutting-machined surface of the KDP-crystal workpiece has a strong anisotropy, and its surface features are mainly composed of profiles in the cutting direction, which will cause waviness to a certain extent. Waviness can exert a significant impact on the surface quality of optical crystals. In severe cases, it may cause dispersion and severely affect the optical performance of optical-crystal components. Therefore, studying the cause of these waviness errors on machined surfaces is of great importance for improving the optical performance of crystals.

\section{Workpiece Detection Signal Processing and Frequency Identification}

The ultra-precision fly-cutting-machined surface of a KDP-crystal workpiece retains machining marks; that is, it has regular periodic components formed from feeding and random components resulting from various variables (e.g., spindle and ambient vibrations) and is a very complex mixed signal. The conventional $R_{q}$ is unable to represent the spatial frequency of the microstructure of such a surface. Especially for KDP crystals, special materials used in the field of nonlinear optics, the various frequencies on the surface will interfere with the characteristics of the laser beam at a specific wavelength. Being able to find a method to describe specific frequency characteristics, especially 
waviness characteristics, is of great significance for studying and evaluating the surface quality of crystal components. For a machined surface, the PSD method can be used to calculate the specific spatial frequency value contained in the surface, and a multiscale wavelet transform can be used to decompose the original surface on multiple scales.

Based on the above analysis combined with the results in Sections 3 and 4 , the features of the fly-cutting-machined surface of the KDP-crystal workpiece consisted mainly of the profiles in the cutting direction. In this study, wavelet and power spectral density methods are used to analyze the cutting direction profile of the machined surface of the KDP crystals in the frequency domain, which lays a theoretical foundation for analyzing the frequency characteristics of the surface microtopography, especially the relationship between the waviness characteristics and various processing factors.

A 1D discrete wavelet transform is performed to analyze the profiles of the machined surface of the KDP-crystal workpiece in Figure 3. Prior to analysis, the signals are filtered to eliminate the effects of the test instruments. The compactly supported orthogonal wavelet base db3 (Third-level Daubechies wavelet) is used to perform five-level decomposition and analysis on the surface frequency information for the KDP-crystal workpiece. Figure 11 shows the reconstructed low-frequency (A1-A5) and high-frequency (D1-D5) signals. As demonstrated in Figure 11a, each low-frequency surface signal is a combination of multiple frequency features and exhibits a topology similar to the original surface profile. Figure $11 \mathrm{~b}$ shows the high-frequency surface information obtained from wavelet decomposition. As demonstrated in Figure 11b, D4 and D5 have relatively large form errors, corresponding to the waves visible on the surface. However, the surface-frequency information obtained from the wavelet method is limited. Therefore, the PSD method is used to further analyze the surface.

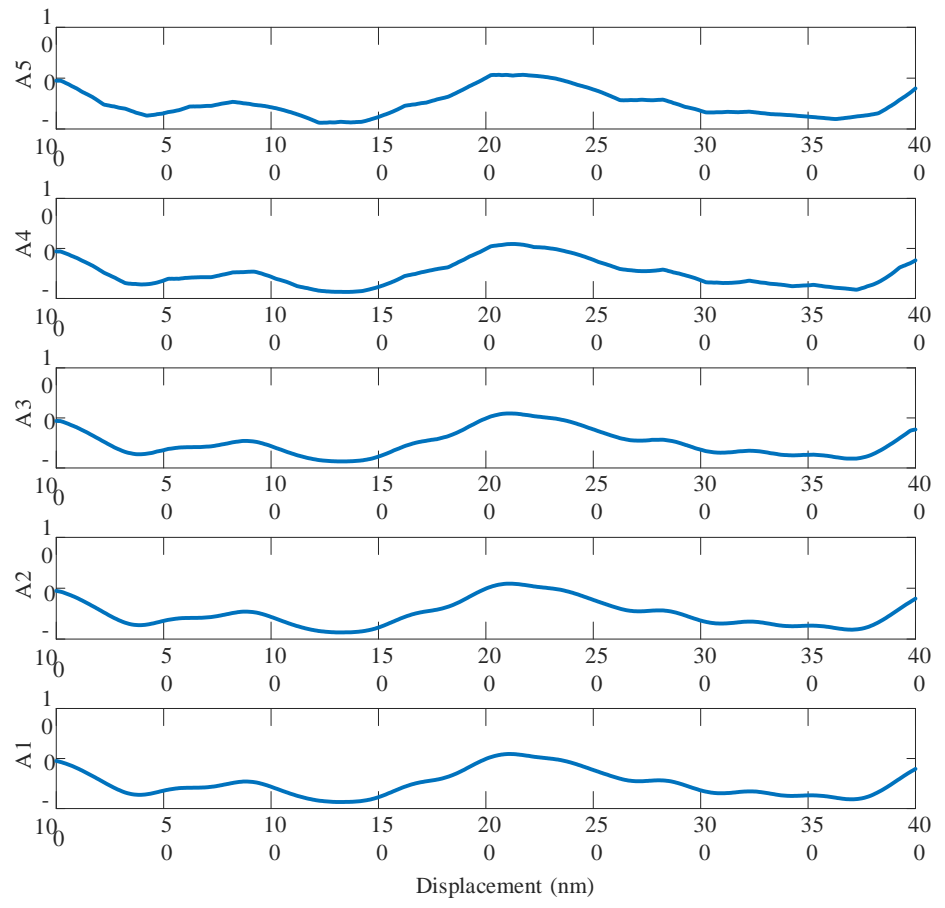

(a)

Figure 11. Cont. 


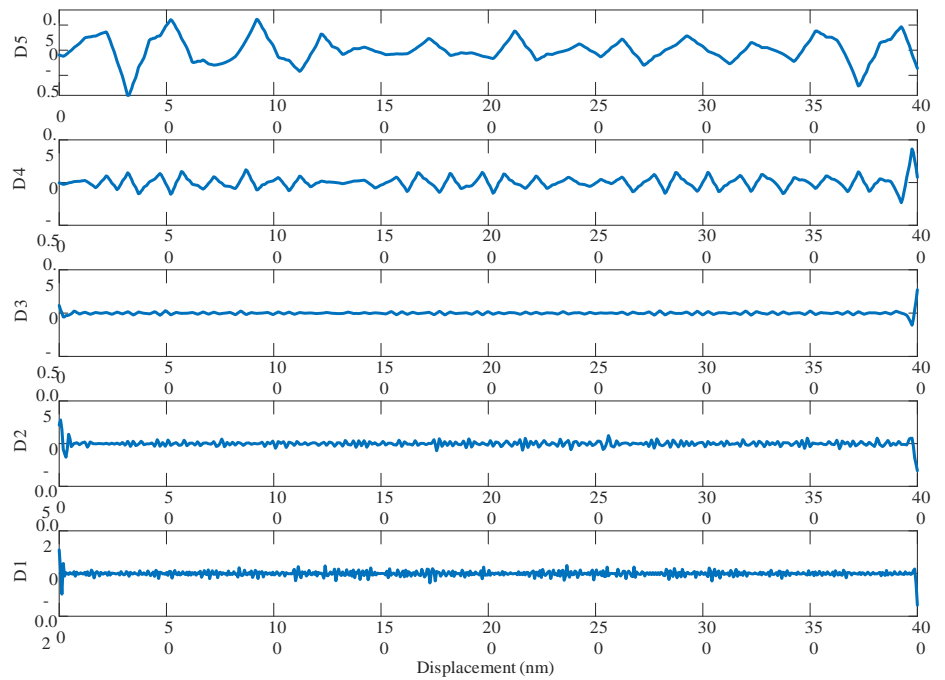

(b)

Figure 11. Wavelet transform decomposition result: (a) Low-frequency signal; (b) High-frequency signal.

The PSD method is used to analyze the PSD of the high-frequency information obtained from wavelet decomposition in Figure 11, as shown in Figure 12. To directly identify the relationship between the surface profile and vibration, Equation (7) is used to transform the high-frequency information obtained by wavelet decomposition for the surface profiles from the spatial domain to the frequency domain [6]:

$$
\omega_{n}=\frac{\pi R \times n \times \cos \theta}{60 T_{D}}
$$

where $\theta$ is the maximum angle of the tangential direction during cutting and $\theta=0^{\circ}, T_{D}$ is the spatial period of surface waviness and $n$ is the frequency of surface waviness.
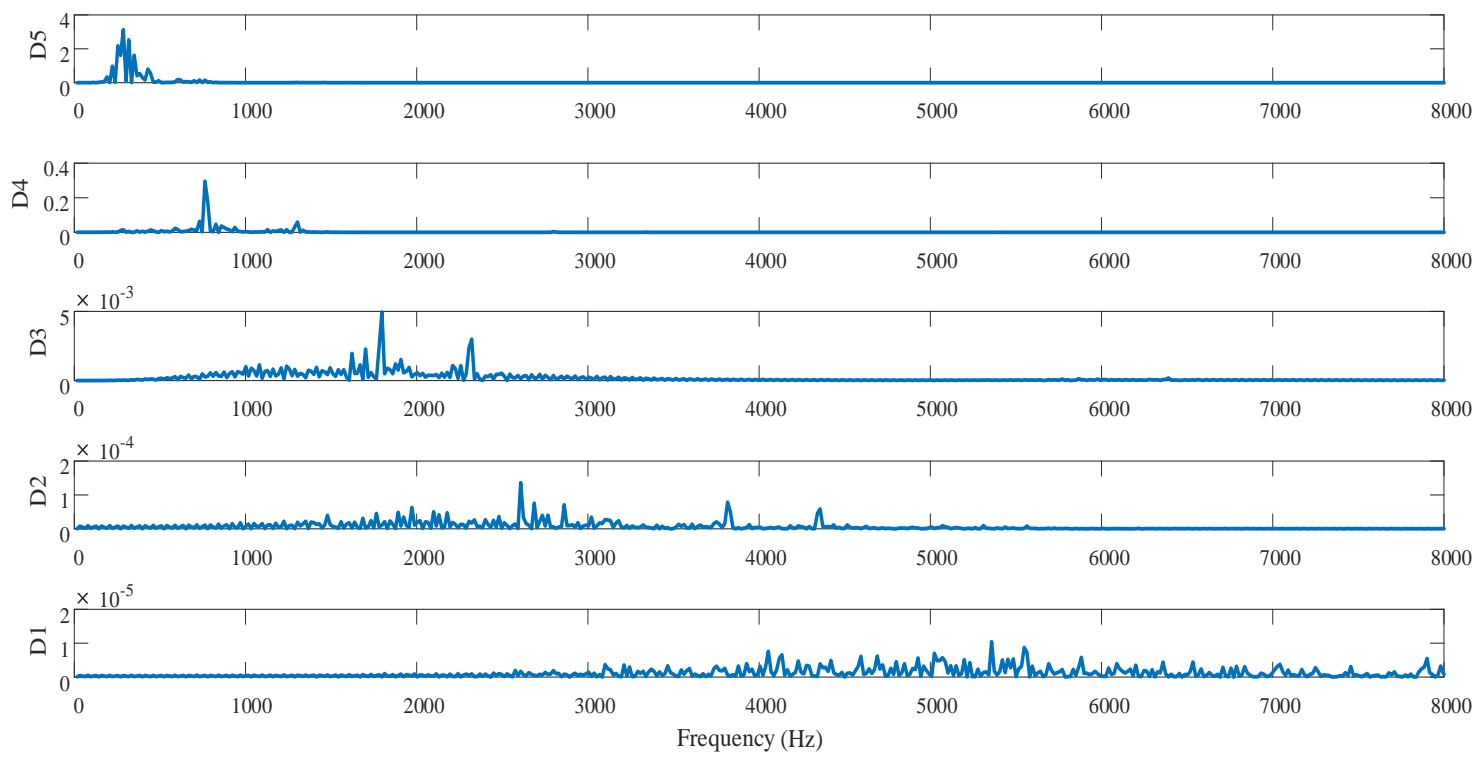

Figure 12. Power spectrum of high-frequency signal after wavelet decomposition.

It can be seen from Figure 12 that the corresponding frequency range is different on each scale, and the variation of the high-frequency PSD on each scale is caused by a specific reason. For the machined surface studied, the frequency components of D1-D4 are mainly related to the material properties and anisotropic cutting parameters and corresponded to relatively high frequency after 
wavelet decomposition. The frequency components of D5 are primarily related to the spindle vibration and were relatively low. To further identify the vibration frequency caused by spindle vibration, the simulated displacement and the PSD of the detail signal D5 in Figure 12 are comparatively analyzed, as shown in Figure 13. As demonstrated in Figure 13, the relatively high-frequency waves (frequency: 270-600 Hz) on the machined surface of the optical KDP-crystal workpiece are caused by spindle vibration.

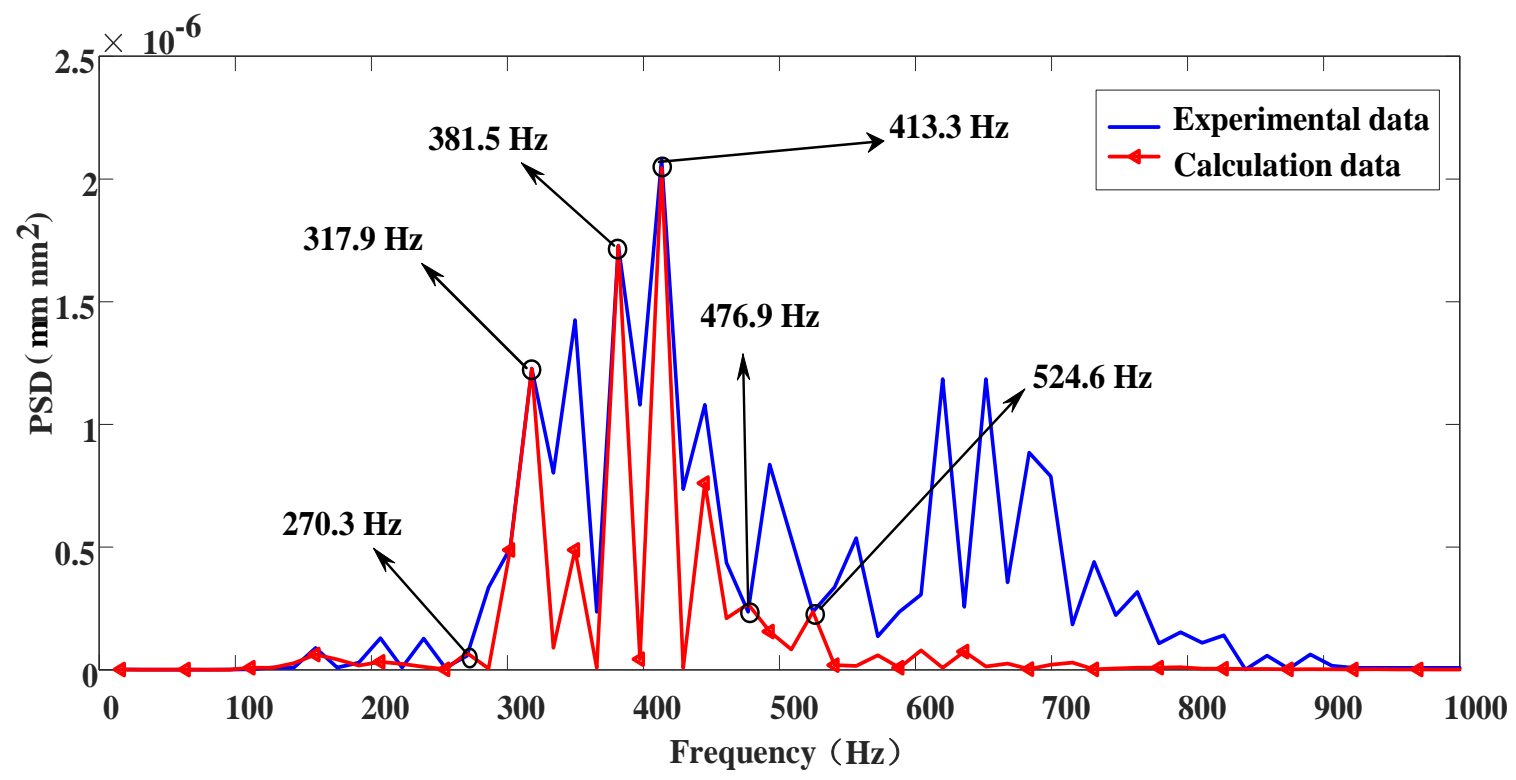

Figure 13. Comparison of PSD between simulation and experimental results.

\section{Conclusions}

In this study, wavelet, fractal, and PSD methods are employed to analyze the machined surface of a KDP-crystal workpiece. Additionally, the relationship between the surface roughness and the fractal dimension during the ultra-precision fly-cutting process is examined. Moreover, the anisotropy of the surface morphology is analyzed. Furthermore, based on the orientation of the machined surface, the cause of surface waviness errors is revealed. The main conclusions derived from this study are summarized as follows:

(1) The fractal and 2D PSD methods are used to calculate and analyze the features of the machined surfaces of various materials. The machined surface of the KDP-crystal workpiece exhibits strong anisotropy, and the features of the surface are mainly composed of contours in the cutting directions.

(2) The wavelet-transform and PSD methods are employed to analyze the tangential waves on the machined surfaces and identify the scale of the anisotropy and spindle vibration for various materials from a frequency-domain perspective. Additionally, the frequency range of the spindle vibration is identified based on the machining experiment. On this basis, the cause of machined surface waviness errors is revealed.

Author Contributions: D.C.: data curation, formal analysis, writing — original draft, writing — review and editing; S.Z.: Formal analysis, methodology, writing—original draft, writing_review and editing. J.L.: investigation, resources, supervision, writing-review and editing. C.Z.: data curation, validation, visualization. R.P.: data curation, validation, visualization. All authors have read and agreed to the published version of the manuscript.

Funding: This research was funded by the National Natural Science Foundation of China Grant (51875005 and 51475010). The National Science and Technology Major Project of China(2016ZX04003001).

Conflicts of Interest: The authors declare no conflict of interest. 


\section{References}

1. Kanimozhi, D.; Nandhini, S.; Indirajith, R. Effects of dyes in the growth, optical, mechanical and dielectric properties of KDP crystals. J. Mater. Sci. 2019, 30, 10244-10255. [CrossRef]

2. Taylor, J.S.; Carlisle, K.; Klingmann, J.L.; Geraghty, P.; Saito, T.T.; Montesanti, R.C. Precision engineering within the national ignition campaign. In Proceedings of the 10th International Conference of the European Society for Precision Engineering and Nanotechnology, Delft, The Netherlands, 31 May-4 June 2010.

3. Adamczak, S.; Zmarzły, P. Influence of raceway waviness on the level of vibration in rolling-element bearings. Bull. Pol. Acad. Sci. Tech. 2017, 65, 541-551. [CrossRef]

4. Costa, R.; Angelico, D.; Reis, M.S.; Ataíde, J.M.; Saraiva, P.M. Paper superficial waviness: Conception and implementation of an industrial statistical measurement. Anal. Chim. Acta 2005, 544, 135-142. [CrossRef]

5. Cao, X.; Lin, B.; Zhang, X. A study on grinding surface waviness of woven ceramic matrix composites. Appl. Surf. Sci. 2013, 270, 503-512. [CrossRef]

6. Chen, W.; Sun, Y. Influence of low-spatial frequency ripples in machined potassium dihydrogen phosphate crystal surfaces on wavefront errors based on the wavelet method. Opt. Eng. 2015, 54, 024101. [CrossRef]

7. Munoa, J.; Beudaert, X.; Dombovari, Z.; Altintas, Y.; Budak, E.; Brecher, C.; Stepan, G. Chatter suppression techniques in metal cutting. CIRP Ann. 2016, 65, 785-808. [CrossRef]

8. Uddin, M.S.; Seah, K.H.W.; Li, X.P.; Rahman, M.; Liu, K. Effect of crystallographic orientation on wear of diamond tools for nano-scale ductile cutting of silicon. Wear 2004, 257, 751-759. [CrossRef]

9. Wang, S.J.; To, S.; Cheung, C.F. An investigation into material-induced surface roughness in; ultra-precision milling. Int. J. Adv. Manuf. Technol. 2013, 68, 607-616. [CrossRef]

10. Zhang, S.J.; To, S. A theoretical and experimental study of surface generation under spindle vibration in ultra-precision raster milling. Int. J. Mach. Tools Manuf. 2013, 75, 36-45. [CrossRef]

11. Chi, F.C.; Lee, W.B. A multi-spectrum analysis of surface roughness formation in ultra-precision machining. Precis. Eng. 2000, 24, 77-87.

12. Chen, G.; Sun, Y.; An, C.; Zhang, F.; Sun, Z.; Chen, W. Measurement and analysis for frequency domain error of ultra-precision spindle in a flycutting machine tool. Proc. Inst. Mech. Eng. Part B J. Eng. Manuf. 2018, 232, 1501-1507. [CrossRef]

13. An, C.H.; Zhang, Y.; Xu, Q.; Zhang, F.H.; Zhang, J.F.; Zhang, L.J.; Wang, J.H. Modeling of dynamic characteristic of the aerostatic bearing spindle in an ultra-precision fly cutting machine. Int. J. Mach. Tools Manuf. 2010, 50, 374-385. [CrossRef]

14. Baek, D.K.; Ko, T.J.; Kim, H.S. Optimization of feedrate in a face milling operation using a surface roughness model. Int. J. Mach. Tools Manuf. 2001, 41, 451-462. [CrossRef]

15. Cheung, C.F.; Lee, W.B. Characterisation of nanosurface generation in single-point diamond turning. Int. J. Mach. Tools Manuf. 2001, 41, 851-875. [CrossRef]

16. Sayles, R.S.; Thomas, T.R. Surface topography as a non-stationary random process. Nature 1978, 271, 431-434. [CrossRef]

17. Yang, X.; An, C.; Wang, Z.; Wang, Q.; Peng, Y.; Wang, J. Research on surface topography in ultra-precision flycutting based on the dynamic performance of machine tool spindle. Int. J. Adv. Manuf. Technol. 2016, 87, 1957-1965. [CrossRef]

(C) 2020 by the authors. Licensee MDPI, Basel, Switzerland. This article is an open access article distributed under the terms and conditions of the Creative Commons Attribution (CC BY) license (http://creativecommons.org/licenses/by/4.0/). 